DOI: http://doi.org/10.46667/renbio.v14i1.458

\title{
IDENTIDADES DE GÉNERO EN LA ENSEÑANZA DE LA BIOLOGÍA: ENTRE LA VIOLENCIA OBJETIFICADORA DE LA CISNORMATIVIDAD Y LOS SABERES TRANS-GRESORES EN SALA DE AULA
}

\author{
GENDER IDENTITIES IN THE TEACHING OF BIOLOGY: BETWEEN THE \\ OBJECTIFYING VIOLENCE OF CISNORMATIVITY AND TRANS-GRESSIVE \\ KNOWLEDGE IN THE CLASSROOM
}

\author{
IDENTIDADES DE GÊNERO NO ENSINO DE BIOLOGIA: ENTRE A VIOLÊNCIA \\ OBJETIFICADORA DA CISNORMATIVIDADE E OS SABERES \\ TRANS-GRESSORES EM SALA DE AULA
}

Yonier Alexander Orozco Marín ${ }^{1}$

\begin{abstract}
Resumen
El objetivo del trabajo fue caracterizar tensiones entre la violencia objetificadora de la cisnormatividad y saberes transgresores, movilizadas durante el desarrollo de una propuesta didáctica para la enseñanza del sistema endocrino, por medio del análisis de los debates alrededor de "identidad de género" en los deportes de alto rendimiento, con estudiantes del grado octavo de la educación básica. Por medio del uso de la autobiografía como registro de la acción política del sujeto investigador/profesor, el trabajo permite reconocer que propuestas didácticas de apariencia progresista/transgresora no están exentas de reforzar violencias en la enseñanza de la biología, y que problematizar la cisnormatividad como estructura de opresión que produce y legitima la transfobia es una tarea importante para el campo.
\end{abstract}

Palabras clave: Educación sexual; Enseñanza de la biología y cisgeneridad; Enseñanza de la biología y transgeneridad; Género y sexualidad; Pedagogías trans.

\begin{abstract}
The objective of the work was to characterize tensions between the objectifying violence of cisnormativity and transgressive knowledge, mobilized during the development of a didactic proposal for the teaching of the endocrine system, through the analysis of the debates around "gender identity" in sports high performance, with students in the eighth grade of basic education. Through the use of autobiography as a record of the political action of the researcher/professor, the work allows us to recognize that didactic proposals with a progressive/transgressive appearance are not exempt from reinforcing violence in the teaching of biology, and that problematizing cisnormativity as structure of oppression that produces and legitimizes transphobia is an important task for the research field.
\end{abstract}

Keywords: Sex education; Teaching of biology and cisgenerity; Teaching of biology and transgenderity; Gender and sexuality; Trans pedagogies.

\footnotetext{
${ }^{1}$ Máster en Enseñanza de las Ciencias y las Matemáticas. Universidad Federal de Acre (UFAC) Rio Branco, AC - Brasil. Estudiante de Doctorado en Educación Científica y Tecnológica - Universidad Federal de Santa Catarina (UFSC). Florianopólis, SC - Brasil. E-mail: apmusicomano@gmail.com
} 


\section{Resumo}

O objetivo do trabalho foi caracterizar as tensões entre a violência objetificadora da cisnormatividade e saberes transgressores, mobilizados durante o desenvolvimento de uma proposta didática para o ensino do sistema endócrino, por meio da análise dos debates em torno da "identidade de gênero" nos esportes de alto rendimento, com alunos da oitava série do ensino fundamental. Por meio do uso da autobiografia como registro da ação política do pesquisador/professor, o trabalho permite reconhecer que propostas didáticas de aparência progressista/transgressora não estão isentas de reforçar violências no ensino de biologia, e que problematizar a cisnormatividade, como estrutura de opressão que produz e legitima a transfobia, é uma tarefa importante para o campo.

Palavras-chave: Educação sexual; Ensino de biologia e cisgeneridade; Ensino de biologia e transgeneridade; Gênero e sexualidade; Pedagogias trans.

\section{Introducción}

Dentro del campo de investigación en enseñanza de las ciencias, particularmente desde la enseñanza de la biología, recientemente se vienen incorporando discusiones relacionadas con asuntos de justicia social. Los debates alrededor de los géneros y las sexualidades y su diálogo con la enseñanza de la biología, no solo como temas a ser presentados, sino también como ejes estructurantes de la propia biología en sus valores, prácticas, discursos y epistemologías, vienen adquiriendo protagonismo en las recientes décadas.

La biología y su enseñanza, en sus versiones más hegemónicas, han estado marcadas por discursos, prácticas, valores y epistemologías que pueden reforzar exclusiones de determinados sujetos, y dotar de cientificidad a preconceptos que colocan a algunos cuerpos en un lugar de normalidad y, que al mismo tiempo, marginalizan otras formas de ser/existir en el mundo, asociándolas a patología, especialmente a los cuerpos trans, travestis y no-binarios. Sandro Santos y Elenita Pinheiros (2019) mencionan que en la enseñanza de la biología se han reforzado discursos que demarcan, y al mismo tiempo, construyen al cuerpo humano en ideas de organización, de clasificación, de representaciones anatómico-fisiológicas y genéticas de lo que puede ser entendido como masculino o femenino. Representaciones sobre el cuerpo desprovistas de contextos culturales y materiales, así como de la propia subjetividad de las y los sujetos que participan del proceso de enseñanza y de aprendizaje.

Como destaca Alice Pagan (2018) el cuerpo humano que circula por la biología escolarizada está más cercano de ser un cadáver, un cuerpo descuartizado, un conjunto de órganos formando un rompecabezas sin emociones, sin placeres, sin dolores, sin relaciones de poder articuladas a él, sin presiones materiales, sin carga de trabajo, sin frustraciones, sin aquello que representa la vida. 
DOI: http://doi.org/10.46667/renbio.v14i1.458

Circula en la biología escolarizada una perspectiva universalizada del cuerpo condicionada por nociones de blanquitud, no discapacidad, poco peso, limpieza, sin hambre, heterosexualidad y cisgeneridad (generalmente masculina) como la versión universal de los cuerpos, aquella normal y esperada, colocando en las márgenes a los cuerpos que más se distancien de esos marcadores (MARIN, 2019). Sin embargo, Sandro Santos y Matheus Martins (2020) destacan que resistencias vienen surgiendo en la enseñanza de la biología a causa de encuentros y espacios formativos que han provocado tensionamientos en las perspectivas biomédicas que desconsideran realidades culturales y materiales. Nuevos abordajes que no están libres de contradicciones y que han permitido crear nuevas posibilidades y caminos, a pesar de que aún están lejos de ser la perspectiva marcante dentro de la realidad de las escuelas.

Thiago Ranniery (2021) apunta que en estos nuevos abordajes circula un imaginario de género como proyectado estrictamente al campo de la cultura y separado de la naturaleza, la cual es colocada como enemigo a superar por el determinismo biológico que se le asocia. Para el autor "nossas concepções de natureza podem ser tão pobres quanto as concepções de gênero da biologia contra os quais viramos nossas acusações” (RANNIERY, 2021, p. 498).

Violencias como el racismo, el machismo, la homofobia y la transfobia hacen parte de la estructura fundante de las sociedades occidentalizadas y colonizadas adquiriendo una apariencia de naturalidad, pero en realidad representan legados instalados y reforzados para garantizar la reproducción de modelos económicos, sociales, políticos, culturales, afectivos y epistémicos que benefician a determinados sujetos, en detrimento de la explotación acelerada de otros seres humanos y de la naturaleza. De esta manera, se entiende que estas violencias pasan a ser abordadas en diversos campos educativos, entre ellos la enseñanza de la biología, no únicamente como actitudes individuales de algunos sujetos, sino como marcas estructurales de las sociedades que denotan una multiplicidad de relaciones de poder sobre los cuerpos, entre ellas las relaciones de poder epistemológicas.

Este trabajo se propone la discusión de una de esas estructuras de poder: la cisnormatividad. La propuesta nace de la preocupación de percibir que recientes abordajes en la enseñanza de la biología, producidos en su mayoría por personas cisgénero, están colocando sus énfasis en las experiencias, saberes, cuerpos y demandas de las personas trans ${ }^{2}$ (GARCIA, LOPES, LOGUERCIO, 2017; PONTES, NETO, 2017; MARIN, OLIVEIRA, 2019; SANTOS, QUEIROZ, 2019; FARIAS, 2020; MENDEZ, 2020). Yonier Marin, Pamela Nunes y Suzani Cassiani destacan que:

\footnotetext{
${ }^{2}$ De aquí en adelante se utilizará la expresión trans para referirse a las experiencias de vida/cuerpos trans, travestis y no-binarios. 
DOI: http://doi.org/10.46667/renbio.v14i1.458

Duas questões são problemáticas quando se pensam os marcadores sociais da diferença na educação. A primeira consiste em pensar estes assuntos no plano individual e do sujeito, como etiquetas que marcam e essencializam uma pessoa, desconhecendo as relações históricas e coloniais que instituíram, naturalizaram e/ou reforçaram estruturas que determinam os lugares dos corpos na nossa sociedade. Dessa maneira, pensa-se que os corpos construídos como "diferentes" nessas relações, devem ser incluídos, higienizados ou aceitos nas estruturas, mas não se questionam as estruturas em si, e muito menos, as combatem. A segunda questão consiste na problematização dos marcadores sociais da diferença, os quais costumam estar direcionados aos corpos construídos como "diversos" ou "diferentes". Por outro lado, corpos que se situam dentro do considerado como "normais" são protegidos e universalizados, e, portanto, sem necessidade de problematização. (MARIN, NUNES, CASSIANI, 2020, p. 227).

¿Por qué priorizamos el abordaje y/o problematización de las experiencias de vida trans en la enseñanza de la biología, y no la cisnormatividad como dispositivo de poder que genera la exclusión y manutención simbólica, cultural e institucional de la transfobia? ¿Cómo el tratamiento crítico de la cisgeneridad y la cisnormatividad en la biología escolarizada nos puede dar luces sobre otros posibles abordajes que no refuercen posibles violencias hacia las personas trans al colocarlas como objetos de estudio o constante eje de problematización?

El objetivo de este trabajo fue caracterizar las tensiones entre la violencia objetificadora de la cisnormatividad y saberes trans-gresores, movilizadas por estudiantes del grado octavo de un Colegio privado de Bogotá (Colombia) durante la implementación de una propuesta didáctica para la enseñanza del sistema endocrino por medio del análisis de los debates alrededor de "identidad de género" en los deportes de alto rendimiento.

\section{Cisgeneridad y Cisnormatividad}

Este texto es escrito por una persona cisgénero ${ }^{3}$. Las personas cisgénero somos aquellas que gozamos del privilegio de ser consideradas por la mirada colonial como "productos de la naturaleza", de ser aquellas que siguen el orden adecuado de la biología, por no desafiar la categoría de sexo biológico que nos fue asignada por el poder biomédicojurídico al nacer. En esta lógica, naturaleza y biológico aparecen como términos/adjetivos dotados de legitimidad para fijar, determinar los lugares de los cuerpos. Las personas cisgénero, en mayor o menor medida, nos sentimos cómodas con esa autoidentificación con el sexo designado y en cierto modo, la reforzamos y la defendemos ${ }^{4}$. La cisgeneridad es una forma de vivir la experiencia de género en relación a las comprensiones de las sociedades occidentales sobre el sexo biológico, macho o hembra. Ya la cisnormatividad, según

\footnotetext{
${ }^{3}$ Marica. Migrante. Mestizo en Colombia, blanco en Brasil.

${ }^{4}$ Pido a la lectora o lector que por favor no realice una lectura moral sobre este texto. El objetivo no es demonizar la cisgeneridad o generar sentimientos de victimización por lo que se es, pues cualquier identidad de género indica contradicciones y matices. El objetivo trata más de denunciar el poder de la cisnormatividad como norma y obligatoriedad de los cuerpos, articulada a otras estructuras de opresión, tal vez reforzadas por la enseñanza de la biología.
} 
DOI: http://doi.org/10.46667/renbio.v14i1.458

Vergueiro (2015) se refiere a "uma série de forças socioculturais e institucionais que discursivamente produzem a cisgeneridade como natural" (VERGUEIRO, 2015, p. 69). Es decir, la cisnormatividad hace referencia a los medios individuales, institucionales, discursivos, afectivos y epistemológicos que refuerzan la cisgeneridad como obligación de los cuerpos, mientras marginaliza y asocia con patología posibilidades fuera de esos moldes.

Viviane Vergueiro (2015) asocia la cisnormatividad como legado colonial articulado con las epistemologías eurocéntricas sobre género. Según la autora:

Considerar a cisgeneridade e a cisnormatividade deve estar atrelado, assim, a uma percepção crítica destes projetos coloniais como limitadores e desumanizadores de um amplo espectro de corpos, identificações e identidades de gênero não normativas, para muito além dos conceitos ocidentalizados de gênero. É importante ter em mente, assim, que as identidades trans*, no geral, são produzidas no interior de contextos ocidentalizados - como, por exemplo, a categoria transexual, produzida a partir dos discursos médicos articulados com epistemologias eurocêntricas. (VERGUEIRO, 2015, p. 48).

En la perspectiva de Marlene Wayar (2007) la cisnormatividad también se refiere a un régimen de miedo. Miedo alimentado por la exposición morbosa y amarillista del cuerpo de la travesti muerta, descuartizada y abandonada en la total impunidad, que instala la cisgeneridad como disfraz de sujeto, cuando en realidad consiste en la teatralización del miedo.

Viviane Vergueiro (2015) correlaciona la cisnormatividad con otros legados coloniales que toman como objetivo el cuerpo y lo moldan a servicio del cis-tema injusto de explotación capitalista colonial, tales como la heterosexualidad, la heteronormatividad y la blanquitud. Así, la cisnormatividad también consiste en la demarcación de los lugares asignados a cuerpos que desafían esos legados, sin considerar las propias percepciones y saberes de esas personas. Es a esto que denomino la violencia objetificadora de la cisnormatividad. Los actos que colocan cuerpos trans como objetos de estudio para ser decodificados a la luz del legado colonial epistémico de la cisnormatividad. Son actos que colocan personas cisgénero en situaciones de poder para estudiar personas trans, definir discursivamente sus lugares y en algunos casos, perpetuar violencias simbólicas y materiales sin tener que asumir responsabilidades o compromisos por esos actos. La impunidad es marca de la cisnormatividad.

Esta es una realidad que ha venido siendo transformada por los propios activismos trans. Acuñar el propio término Cis es producto de estas luchas (VERGUEIRO, 2015). Sara York, Megg Oliveira y Bruna Benevides (2020) son enfáticas en que es urgente desnaturalizar la violencia objetificadora de la cisnormatividad en espacios académicos y educativos. Las autoras en su Manifiesto Travesti destacan "Não falem de nossos nós, por nós e/ou sem nós!" (p. 8). Y complementan con "podemos escrever juntas/os/es/xs. Respeite o lugar de fala - fale a partir do que lhe toca e como lhe toca - isso significa ir além e (re)pensar (a sua pesquisa, seu objeto) seu olhar sobre a vida" (p. 9). 
DOI: http://doi.org/10.46667/renbio.v14i1.458

La investigación de Viviane Vergueiro (2015) ofrece tres categorías analíticas que ella denomina trazos de la cisgeneridad y que pueden contribuir a reconocer las características de las vivencias corporales que la cisnormatividad obliga y defiende. Son estos tres trazos: La pre-discursividad, la binariedad y la permanencia (VERGUEIRO, 2015). La autora los define de la siguiente manera:

- La pre-discursividad se refiere al entendimiento socio-cultural, históricamente normativo de que es posible definir sexos/géneros de seres a partir de criterios objetivos y de ciertas características corporales, independientemente de cómo sean sus autopercepciones o de las posiciones en contextos interseccionales y socioculturales en que estén localizadas. La pre-discursividad es un trazo cisnormativo instrumentalizado por el poder-saber atribuido a especialistas, y que se encarna en violencias médicas y procedimientos quirúrgicos no consentidos;

- La binariedad comprende que si es posible definir objetivamente los géneros (prediscursividad), cuerpos/seres "normales" son viables mientras tengan géneros definidos a partir de dos, y solamente dos, alternativas: macho/hombre, hembra/mujer;

- La permanencia es la premisa asumida de que cuerpos ideales presentan coherencia fisiológica y psicológica en términos de sus pertenencias a una categoría de sexo biológico y que esas coherencias deban ser expresadas de manera consistente a través de la vida de una persona, identificando como patología cualquier desvío.

A pesar de que la autora no lo mencione explícitamente, considero que estos trazos pueden verse reforzados por los discursos hegemónicos de la biología que circulan en la educación, no solo en referencia a la especie humana, sino también en otras especies. De esta manera, podríamos pensar si el problema está realmente en las concepciones de género de la biología contra las cuales hemos colocado nuestras acusaciones o si es necesario que vayamos más a fondo y reconozcamos la cisnormatividad como estructura de opresión, que alineada con otras, ocasiona que la biología, así como otros saberes, operen de esas maneras en los campos investigativos y educativos.

Bruno Tavares (2018) destaca que los libros de biología refuerzan la cisnormatividad, tanto en las imágenes de los cuerpos que presentan, así como en sus representaciones escritas sobre conceptos de sexo y género siempre en perspectivas binarias. Sin embargo, esto no puede llevarnos a pensar que al incluir otras representaciones semióticas en estos materiales el problema de la cisnormatividad desaparece. No se trata de completar inventarios de la diversidad, y si de visibilizar y problematizar la estructura opresiva de la cisnormatividad en diferentes espacios de la vida y en las propias construcciones de identidad de género de las personas que participamos del proceso escolar. 
DOI: http://doi.org/10.46667/renbio.v14i1.458

De esa manera, no basta con suponer que lo cis está ahí de manera implícita, presente en los procesos de enseñanza y aprendizaje, es necesario marcarlo explícitamente para problematizar sus impactos sobre lo que aprendemos, sobre lo que decimos, sobre lo que pensamos de nosotras y nosotros mismos, sobre las personas que hemos considerado "otras", sobre la naturaleza y la vida. La cisgeneridad y la cisnormatividad aun han sido poco problematizadas en la enseñanza de la biología ${ }^{5}$, por lo menos de manera explícita.

Es necesario estar atentas y atentos a las articulaciones de la cisnormatividad con otras estructuras coloniales. En las sociedades latinoamericanas, tan profundamente marcadas por el racismo estructural y el capitalismo como modelo económico, contemplar un diálogo con la categoría de blanquitud es indispensable (NASCIMENTO, 2020). Una mujer cisgénero negra no es necesariamente privilegiada en su identidad de género. Pues la blanquitud también demarca espacios institucionales, sociales e individuales para garantizar la instalación y actualización del proyecto capitalista y colonial que coloca a las mujeres negras e indígenas (cis y trans) en la base de la pirámide productiva, con una violación excesiva e intencionada de sus derechos. Atribuyendo al mismo tiempo, privilegios simbólicos, y en algunos casos materiales, a las personas blancas.

\section{Enseñanza de la biología y las identidades de género trans ¿Y también la cis?}

En la tabla 1 se presentan algunos trabajos ${ }^{6}$ que articulan propuestas didácticas para la enseñanza de la biología y la química abordando los saberes, experiencias, cuerpos y/o luchas de las personas trans. Son trabajos recientes en el campo y han permitido dialogar con una realidad hasta ahora bastante desconsiderada, debido al discurso y pánico moral que caracteriza a la escuela cuando se encuentra con cuerpos disidentes de las normas sexuales y de género

\footnotetext{
${ }^{5}$ Como ejemplo, en uno de los trabajos leídos para fundamentar este texto, que abordaba la enseñanza de la biología asociada a las cuestiones de la transexualidad, encontré que la marcación de la identidad trans aparece más de treinta veces en el texto, pero la marcación cis no aparece ni una sola vez, ni siquiera para hacer referencia a las propias personas que escribieron el texto.

${ }^{6}$ No se trata de una revisión exhaustiva o de estado de arte. Se tratan de algunos antecedentes para la reflexión y construcción de este trabajo.
} 
DOI: http://doi.org/10.46667/renbio.v14i1.458

Tabla 1. Algunos trabajos que articulan enseñanza de la biología/química con las identidades de género trans. Siglas: (ERV: Como experiencia y/o relato de vida) (RTM: Como referencial teórico y/o metodológico).

\begin{tabular}{|c|c|c|c|c|c|}
\hline \multirow[t]{2}{*}{ Titulo/Ano } & \multirow[t]{2}{*}{$\begin{array}{l}\text { Temática de la } \\
\text { biología/química } \\
\text { abordada }\end{array}$} & \multirow[t]{2}{*}{$\begin{array}{l}\text { Referencias } \\
\text { de personas } \\
\text { trans }\end{array}$} & \multicolumn{2}{|c|}{$\begin{array}{c}\text { Marcación de } \\
\text { la identidad } \\
\text { de género }\end{array}$} & \multirow[t]{2}{*}{$\begin{array}{l}\text { Diálogo con otras } \\
\text { categorías de } \\
\text { opresión }\end{array}$} \\
\hline & & & Cis & Trans & \\
\hline $\begin{array}{l}\text { "Uma canção pra você" a } \\
\text { música em uma proposta de } \\
\text { sequência didática voltada } \\
\text { para um ensino de biologia } \\
\text { não sexista. } 2020\end{array}$ & Genética & $\begin{array}{l}\text { ERV } \\
\text { RTM }\end{array}$ & $\mathrm{Si}$ & $\mathrm{Si}$ & Raza/Etnia \\
\hline $\begin{array}{l}\text { La perspectiva de género en } \\
\text { la enseñanza del sistema } \\
\text { endocrino: una experiencia } \\
\text { didáctica en contexto de } \\
\text { educación remota. 2020 }\end{array}$ & Sistema endocrino & No & No & $\mathrm{Si}$ & No \\
\hline $\begin{array}{l}\text { Problematizando as relações } \\
\text { entre química-biologia e } \\
\text { questões de gênero: } \\
\text { Possibilidades e desafios na } \\
\text { Educação de Jovens e } \\
\text { Adultos. 2019 }\end{array}$ & Sistema endocrino & $\begin{array}{l}\text { ERV } \\
\text { RTM }\end{array}$ & $\mathrm{Si}$ & $\mathrm{Si}$ & $\begin{array}{l}\text { Género, raza/etnia, } \\
\text { nacionalidad, clase } \\
\text { social. }\end{array}$ \\
\hline $\begin{array}{l}\text { A fada madrinha da } \\
\text { passabilidade: hormônios e o } \\
\text { ensino de química. } 2017\end{array}$ & Sistema endocrino & ERV & $\mathrm{Si}$ & $\mathrm{Si}$ & Heteronormatividad \\
\hline $\begin{array}{l}\text { Trans-orgânica: uma } \\
\text { proposta de ensino de } \\
\text { química orgânica utilizando } \\
\text { as temáticas de gênero e } \\
\text { sexualidade. } \mathbf{2 0 1 7}\end{array}$ & $\begin{array}{c}\text { Funciones orgánicas } \\
\text { y hormonas }\end{array}$ & RTM & No & $\mathrm{Si}$ & No \\
\hline
\end{tabular}

Fuente: Autor

Son trabajos que obligan a nuevos abordajes y a la introducción de nuevos conceptos como el de la pasabilidad (PONTES, NETO, 2017), discusiones sobre las limitaciones de nuestros entendimientos sobre sexo biológico (FARIAS, 2020); estereotipos sociales sobre el papel de las hormonas en la feminidad y masculinidad (GARCIA, LOPES, LOGUERCIO, 2017; MARIN, OLIVEIRA, 2019) y la salud de las personas trans (MENDEZ, 2020).

Algunos de estos trabajos pueden estar alineados con el refuerzo de una violencia objetificadora de la cisnormatividad cuando las personas trans aparecen únicamente como cuerpos a ser estudiados o problematizados en la propuesta didáctica. Silenciar las posibilidades de considerar otras posibilidades de vivir la corporalidad, la identidad de género y la sexualidad, puede ser tan violento cuanto abordarlas como objeto de estudio, sin el diálogo necesario con las experiencias de vida y saberes que esos cuerpos encarnan. Sin embargo, en la mayoría de propuestas las personas trans también aparecen como referentes para el trabajo, y en algunos casos, inclusive como revisoras de las propuestas planteadas (FARIAS, 2020). 
DOI: http://doi.org/10.46667/renbio.v14i1.458

En algunos casos las personas trans entran como referentes por medio de relatos y experiencias de vida (ERV) para ser leídos y analizados por las y los estudiantes a quienes se dirige la propuesta, pero no siempre como referentes teóricos y metodológicos de la investigación y/o propuesta (RTM). Este es un aspecto a tener cuidado, pues un trazo de la violencia objetificadora de la cisnormatividad, así como de la blanquitud, es suponer que aquel que se ha construido como el "otro" (la personas trans, la persona racializada), puede en lo máximo contribuir con experiencias y relatos, para que sean teorizados y legitimados por las miradas cisgénero y blancas de los contextos académicos. Pensemos en lo potente que sería que estudiantes cis pudiesen analizar sus propios relatos y experiencias de vida sobre las construcciones e imposiciones de sus cisgeneridades, a la luz de teorías, conceptualizaciones y saberes trans.

En algunos trabajos la cisgeneridad es marcada responsablemente, inclusive para referirse a los propios autores de los trabajos (MARIN, OLIVEIRA, 2019; FARIAS, 2020). Sin embargo, la cisnormatividad como concepto no aparece explícitamente problematizada, aunque puede inferirse que de manera implícita algunos trazos de la cisgeneridad (VERGUEIRO, 2015) puedan ser problematizados en las propuestas. Algunos trabajos proponen diálogos con otras categorías de opresión en sus propuestas didácticas, aunque no siempre de manera tan explícita, lo cual igualmente refuerza la norma al considerar que ésta no debe ser enunciada.

Tal vez un paso para reaproximarnos a una mirada más creativa sobre la diversidad sexual y de género en la enseñanza de la biología, no está en profundizar esos caminos que objetifican y problematizan qué es lo trans. Inclusive porque las propias experiencias de vida trans son diversas. No todas por ejemplo pasan por procesos de hormonización. Pero aun así las propuestas didácticas alrededor de las hormonas y el sistema endocrino son las más comunes. El consumo de hormonas puede ser más común en mujeres cis como método anticonceptivo, o en hombres cis por el consumo del viagra y/o anabolizantes en los gimnasios, que en las mismas personas trans. Propuestas que estimulen pensar como los juegos discursivos de la biología se tejen en medio de la defensa de la cisnormatividad (articulada a la blanquitud, heterosexualidad...) pueden ser más interesantes, en diversos temas de la biología, no solo aquellos relacionados con el cuerpo. Las producciones de intelectuales trans no se reducen a discusiones sobre el cuerpo, inclusive dentro de la enseñanza de la biología encontramos los trabajos de Alice Pagan (2020) que insisten en el ecotransfeminismo como posibilidad para estimular relaciones ecosociales, encuentros más afectivos y viscerales con la naturaleza y no únicamente desde la racionalidad.

El desafío aún pendiente es el de realizar investigaciones que permitan registrar y sistematizar los aprendizajes y problematizaciones construidas por los estudiantes con la implementación de estas propuestas didácticas. Hasta ahora las investigaciones han estado en el nivel de discusiones teóricas y la planeación de propuestas. Faltan las voces de las y los estudiantes, la voz de la escuela, que tal vez pueda apuntarnos otros caminos. 


\section{Procedimientos Metodológicos}

\subsection{Contexto}

Este texto tiene como propósito revelar algunas tensiones entre violencias objetificadoras de la cisnormatividad y la influencia de saberes trans en medio de una intervención didáctica con estudiantes de grado octavo de la educación básica secundaria (ensino fundamental II no Brasil), de una escuela particular de Bogotá (Colombia) de renta baja/media. Las y los estudiantes participantes fueron 52, agrupados en dos cursos cada uno de 26 estudiantes.

Las y los estudiantes se autoidentifican como hombres cis $(\mathrm{N}=29)$ y mujeres cis $^{7}$ $(\mathrm{N}=23)$, en su totalidad se identificaron como mestizos y/o blancos y tienen edades entre los 13 y 15 años. La escuela donde la intervención fue realizada nunca ha contado con la presencia de personas trans como profesoras, directivas o estudiantes. Las y los estudiantes manifestaron no haber abordado temáticas de identidad de género en otros años escolares o espacios académicos. El autor de este texto fue también el profesor de biología de las y los estudiantes, quien realizó la intervención didáctica al inicio del año escolar durante febreromarzo del 2020.

Debo reconocer que este texto está escrito al revés. Me refiero a que algunas de las reflexiones hasta aquí presentadas son derivadas de la propia experiencia que ahora presentaré. La necesidad de problematizar la cisnormatividad se fundamenta precisamente al haber construido y aplicado la propuesta reproduciendo la violencia objetificadora de la cisnormatividad y encontrar las limitaciones de ese abordaje con mis estudiantes.

\subsection{Propuesta didáctica}

La propuesta fue proyectada para ser aplicada durante ocho sesiones con cada curso, cada una de duración de dos horas y fue inspirada en un conjunto de actividades ya sugeridos por Yonier Marin y Maira Oliveira (2019). Como obligación curricular de la institución, el primer contenido que aparecía para ser abordado con los grados octavos era el sistema endocrino, de esa manera se articuló la propuesta con esos contenidos.

La fundamentación metodológica que acompañó la propuesta didáctica fue el enfoque de estudios de caso, los cuales:

\footnotetext{
${ }^{7}$ En realidad, el reconocimiento de la identidad de género cis ocurrió durante el desarrollo de la propuesta, pues al inicio de la propuesta las y los estudiantes se autorreconocían solo como hombres o mujeres.
} 
DOI: http://doi.org/10.46667/renbio.v14i1.458

Apresentam um cenário para a aprendizagem que espelha a vida real, na qual decisões são tomadas e conclusões feitas com base em informações parciais, inclinações ideológicas e premissas sobre o que se sabe ou não. Isso força os usuários de estudos de caso a assumirem pressupostos sobre o pensamento implícito ou os antecedentes de um episódio, da mesma maneira que fazem quando se defrontam com informações imperfeitas na vida real. (GRAHAM, 2010, p. 25-26).

Fue propuesto a las y los estudiantes un caso de la vida real, sobre el cuál debían posicionarse al inicio y final de la propuesta, aprovechando las actividades realizadas para complejizar (o no) su posicionamiento y fundamentarlo desde diversos argumentos. El caso abordado fue el de Tiffany Abreu, jugadora trans del voleibol profesional femenino en Brasil, sobre el cual se ha movilizado una exagerada polémica en medios de comunicación y de la población en general, sobre la participación o no de personas trans en deportes de alto rendimiento, especialmente de mujeres trans en equipos femeninos.

Para aquel momento, comprendiendo que muchos de los argumentos que involucraban a la ciencia en el caso se relacionaban con el sistema endocrino, me pareció importante traerlo por: a) ser un caso de la vida real, y que podía permitir articular discusiones sobre identidad de género, b) traer el sexo biológico como territorio de poder para los cuerpos y que ha cambiado a lo largo de la historia de los deportes de alto rendimiento, c) por ser un caso con potencial didáctico para promover discusiones y elaboración de argumentos. Las actividades ${ }^{8}$ (tabla 2) se articularon para que las y los estudiantes, asumiendo de manera ficticia el papel del comité olímpico pudieran construir aprendizajes, reflexiones y argumentos sobre el caso. Mi preocupación como profesor no fue que decidieran sí o no sobre el caso, y si, la riqueza de las discusiones y problematizaciones en el proceso.

\footnotetext{
${ }^{8}$ Una descripción más profundizada sobre las actividades de la propuesta didáctica fue socializada en la5 $5^{\mathrm{a}}$ edição do Curso de Atualização para Professoras(es) de Ciências e Biologia - Diálogos entre Biologia e Educação no cenário pandêmico do grupo LIQUENS, da UERJ, disponible como video de youtube en el enlace https://www.youtube.com/watch?v=tKhyopYSIOA (26/04/2021).
} 
DOI: http://doi.org/10.46667/renbio.v14i1.458

Tabla 2. Actividades desarrolladas en la propuesta didáctica.

\begin{tabular}{|c|c|}
\hline Actividad & Descripción \\
\hline 1. Parecer inicial & $\begin{array}{l}\text { Asumiendo el papel de Comité Olímpico, estudiantes emitieron parecer justificado } \\
\text { desde sus sentidos previos sobre el caso de Tiffany }\end{array}$ \\
\hline $\begin{array}{l}\text { 2. Reconociendo el } \\
\text { debate en los medios de } \\
\text { comunicación }\end{array}$ & $\begin{array}{l}\text { Estudiantes elaboraron fanzines con reportajes encontrados en internet sobre el caso } \\
\text { de Tiffany, y los socializaron entre ellos }\end{array}$ \\
\hline $\begin{array}{l}\text { 3. Construcción de } \\
\text { siluetas propias }\end{array}$ & $\begin{array}{l}\text { En pliegos de cartulina las y los estudiantes plasmaron las siluetas de sus cuerpos } \\
\text { con ayuda de colegas. Se realizó una reflexión sobre la diversidad de cuerpos y los } \\
\text { propios imaginarios que ellos tenían sobre sus cuerpos al observar las siluetas }\end{array}$ \\
\hline $\begin{array}{lr}4 . & \text { Identificación } \\
\text { glándulas } & \text { sistema } \\
\text { endocrino y hormonas }\end{array}$ & $\begin{array}{l}\text { Cada estudiante recortó y pegó en las siluetas corporales construidas las glándulas } \\
\text { del sistema inmune con sus respectivos nombres. Se realizó una reflexión sobres las } \\
\text { ideas de masculino y femenino en los cuerpos asociadas a gónadas y hormonas, y } \\
\text { algunos estereotipos difundidos }\end{array}$ \\
\hline $\begin{array}{l}\text { 5. Lectura y discusión de } \\
\text { artículo científico }\end{array}$ & $\begin{array}{l}\text { Se realizó lectura colectiva de artículo científico }{ }^{9} \text { que aborda cambios biológicos en } \\
\text { personas trans después de un año de tratamiento hormonal. Se discutieron conceptos } \\
\text { de sexo biológico, identidad de género y orientación sexual. El profesor explicó el } \\
\text { funcionamiento y niveles de diversas hormonas relatadas en el texto. }\end{array}$ \\
\hline $\begin{array}{l}\text { 6. Debate de video de } \\
\text { Youtube }\end{array}$ & $\begin{array}{l}\text { Grupalmente fue observado el video de Youtube titulado: “¿Es injusto dejar } \\
\text { competir a atletas trans en los deportes de mujeres? | Diagno-Cis 105" del canal de } \\
\text { Youtube Diagno-cis, de la youtube trans mexicana Ophelia Pastrana. }\end{array}$ \\
\hline $\begin{array}{l}\text { 7. Análisis de la historia } \\
\text { del sexo biológico en } \\
\text { deportes de alto } \\
\text { rendimiento }\end{array}$ & $\begin{array}{l}\text { Análisis de casos diversos e históricos en la definición de sexo biológico en } \\
\text { deportes de alto rendimiento y sus intersecciones con categorías como raza, } \\
\text { nacionalidad... Los casos discutidos fueron: María Joaquina Cavalcanti Reikdall } \\
\text { (Patinaje); María Patiño (Atletismo); Caster Semenya (Atletismo); Michael Phelps } \\
\text { (Natación); Patricio Manuel (Boxeo); Edinanci Silva (Judo). }\end{array}$ \\
\hline $\begin{array}{l}\text { 8. Diálogo con persona } \\
\text { experta. }\end{array}$ & $\begin{array}{l}\text { Video conferencia con Ale Mujica Rodríguez, Medique, Doctore en salud colectiva } \\
\text { y personas trans no binaria. Estudiantes prepararon preguntas y las resolvieron } \\
\text { directamente con la persona conferencista. }\end{array}$ \\
\hline 9. Parecer final & $\begin{array}{l}\text { Estudiantes de manera individual realizaron nuevamente parecer argumentado sobre } \\
\text { el caso de Tiffany asumiendo el papel de Comité Olímpico. }\end{array}$ \\
\hline
\end{tabular}

\section{Fuente: Autor}

Trazos de la violencia objetificadora de la cisnormatividad están reforzados desde la propia propuesta didáctica, a pesar de que en algunas actividades se incluyera la escucha y diálogo con especialistas trans sobre el caso estudiado. A final de cuentas, la propuesta obligaba a la ya tan conocida escena de un conjunto de personas cis ejerciendo el poder de estudiar y definir el lugar y posibilidades de personas trans en la sociedad, abriendo espacios al morbo, la exotización y otras manifestaciones simbólicas de violencia.

\footnotetext{
${ }^{9}$ Texto: WIKK, Ana; et al. Muscle Strength, Size, and Composition Following 12 Months of Gender-affirming Treatment in Transgender Individuals. The Journal of Clinical Endocrinology \& Metabolism, v. 105, n. 3, 2020.
} 


\subsection{Construcción y análisis de datos}

Aunque la recepción de la propuesta por parte de las y los estudiantes fue positiva, no necesariamente lo fue para las directivas y/o familias de éstos. De las y los 52 estudiantes participantes, las familias de 29 autorizaron, por medio de consentimiento esclarecido firmado, el uso de los escritos producidos por las y los estudiantes para ser analizados de acuerdo a los objetivos de esta investigación, realizando un tratamiento ético de los datos y manteniendo sigilo sobre los datos de identidad. Además de estos escritos, y siguiendo la propuesta metodológica de autoetnografía de Silvio Santos (2017), realicé registro de mi propia experiencia como profesor en un diario de campo, donde, sin seguir formatos específicos, me permitía colocar relatos, emociones y reflexiones vividas durante la implementación de la propuesta, no solo a nivel de las clases, mas también en episodios vividos con directivas, otros profesores y familias.

Para Silvio Santos (2017) la autoetnografía, como relato biográfico individual, "é um meio para melhor caracterizar a situação de trabalho da perspectiva dos agentes que dela participam" (p. 216). El autor destaca que la autoetnografía permite pensar el papel político de un autor en relación a un tema, la influencia de ese autor en las decisiones y direccionamientos investigativos y sus posibles avances. Es decir, no se trata únicamente de un "método" para la colecta de datos, sino de un registro del accionar político del sujeto autor de la investigación, en este caso, también profesor participante y direccionador de la propuesta.

Se seleccionaron fragmentos de los registros del diario de campo, y de los escritos de las y los estudiantes que autorizaron su participación, en los cuales fuera posible identificar las tensiones entre la violencia objetificadora de la cisnormatividad vivenciada durante la implementación de la propuesta, a partir del reconocimiento de los tres trazos de la cisgeneridad propuestos por Viviane Vergueiro (2015). También fragmentos que permitieran identificar la potencia e influencia de los saberes de personas trans que participaron durante la implementación, sea por medio del diálogo directo con las y los estudiantes, o por medio de la lectura de sus producciones. Nombres ficticios fueron utilizados para identificar las personas mencionadas o autoras de los fragmentos. 


\section{Resultados y discusión}

\subsection{Trazos de la cisnormatividad en acción durante la experiencia}

Uno de los momentos de la implementación donde elementos de la violencia objetificadora de la cisnormatividad se manifestaron de manera más clara, fue en la actividad 2 , de reconocimiento del debate en medios de comunicación y en la cual las y los estudiantes socializaron sus fanzines. De ese momento rescato el siguiente fragmento:

Con 8 A fue un poco más denso. Cuando vieron los fanzines de Tiffany mencionaron "tiene voz de hombre", "es bonita", "está buena", "parece mujer". Juan (estudiante) mencionó explicitamente que le tiene miedo a las personas trans. Con $8 B$ fue más difícil aun, "Está buena, yo la veo en la calle y la volteo a mirar y le hago la vuelta, no parece man" llegué a escuchar, se centraron mucho en la figura de Tiffany antes de su transición, y en ese caso los comentarios fueron más incisivos: "Uy esas patas" "Tremendo gurre que era de hombre" "Mire care nalga" "¿Tendrá labios de chocha?" "¿Qué es que se quiere volver como Tiffany? "No es lo mismo una bola negra que una negra en bola"... (Registro en diario de сатро).

Una serie de calificativos peyorativos, estereotipos y juicios se activaron por parte, especialmente, de los hombres cis del salón. Sabemos que estos pensamientos están presentes en la sociedad, pero lo que llamó mi atención, fue que a pesar de que estos comportamientos no los habían manifestado antes en mi clase, de alguna manera la actividad los hizo sentirse en una situación de poder para descargar un lenguaje tan violento. Mi sospecha es que la propia propuesta de la actividad dotó de legitimidad el accionar violento de la cisnormatividad, a pesar de que la intención fue otra.

Marlene Wayar (2007), Viviane Vergueiro (2015), Sara York, Megg Oliveira y Bruna Benevides (2020) destacan que la mirada cisgénero de los medios de comunicación ha sido una de las armas más violentas hacia las personas trans, pues pocas veces resaltan las luchas, logros o demandas de éstas. Por el contrario, utilizan un lenguaje morboso y explotador de sus cuerpos y pasados. Promover que mis estudiantes trajeran aquello que encontraron en medios de comunicación, sin una preparación previa o mirada crítica, alimentó la relación de poder en la cual la cisnormatividad coloca a personas cis.

Si bien, podría destacar un potencial pedagógico y didáctico de que mis estudiantes expresen sus ideas para que después estas sean problematizadas y discutidas, como de hecho ocurrió, el problema, además la expresión de esas ideas en sí mismas, es reforzar la estructura de poder que autoriza determinados sujetos a posicionarse así delante de otros.

De hecho, en esa misma clase inicié una discusión sobre la misoginia y sobre las formas en que nos han educado de tal manera que, especialmente hombres cis, nos sentimos en derechos de vigilar y opinar sobre el cuerpo de las mujeres. Algunas estudiantes del salón, aunque de manera tímida, manifestaron que no se estaban sintiendo cómodas escuchando esos 
DOI: http://doi.org/10.46667/renbio.v14i1.458

comentarios. Mi silencio inicial también fue cómplice. Es importante resaltar que los comentarios de mis estudiantes no deben ser leídos como patologías morales individuales, y si, como expresiones de violencias estructurales históricamente reforzadas. Inclusive, profesores que nunca se habían acercado a conversar conmigo, a causa de esta propuesta didáctica en acción se acercaron a manifestar ciertas preocupaciones:

El profesor Ángel en sala de profesores cuando escuchó que yo hablaba con otro profesor sobre las actividades con octavo del caso de Tiffany, me dijo: "Profe toca que no los desconstruya tanto porque después empiezan a cuestionar mucho, por ejemplo, a no querer usar uniforme, o a molestar más en clase" (Fragmento del diario de campo)

Le dije al profesor que esa era la idea. La circulación de una propuesta abordando esta situación por la escuela es suficiente para activar el pánico moral de una posible contaminación al buen comportamiento por traer la diversidad. Un miedo a desbaratar el orden linear del discurso educativo. De la experiencia derivo que un cuarto trazo que podemos reconocer de la cisgeneridad, o que tal vez ya está implícito en los tres resaltados por Viviane Vergueiro (2015) es el de la dualidad vigilancia/fragilidad, como imposición y pulsión constante a vigilarse y vigilar comportamientos, cuerpos, maneras a fin de mantenerlas encuadradas dentro de los preceptos de la cis-heteronormatividad, al parecer bastante quebrantables por el miedo que expresan al fantasma de la "diversidad contaminadora".

A pesar de que no hizo parte explícita de las actividades abordando sistema endocrino, en medio de la propuesta en la escuela realizamos un evento al que llamamos el encuentro de géneros. Fue un día en el cual se reunieron en la institución activistas de los feminismos y derechos LGBTI. Para los grados octavos se organizó una charla entre las y los estudiantes con un grupo de cuatro activistas cis y una activista trans. Sobre este momento destaco los siguientes fragmentos:

\begin{abstract}
Al final del encuentro, un estudiante se me acercó y agradeció la actividad, y de manera respetuosa me dijo: "Bien por la actividad, pero no estoy de acuerdo porque uno debería respetar la forma en que lo criaron”. Además algunas informaciones me llegaron de algunas familias. Los papás de Michael fueron al colegio a decir que no querían a su hijo escuchando eso. Le pregunté directamente a Michael que pensaba él, y dijo que para él eso es una huevonada, inventos de la gente, modas por ahí. Igualmente Juan me dijo que su papá le manifestó que no había problema con la actividad pero que se cuidara en caso de que esas personas invadieran su espacio. (Fragmento del diario de campo)
\end{abstract}

El comentario "respetar la forma en que lo criaron" pone de manifiesto la defensa de la permanencia (VERGUEIRO, 2015) como trazo característico de la cisgeneridad. A pesar de que eran varios activistas tocando en temas polémicos tales como la lucha indígena, el machismo, e inclusive, la historia del movimiento de liberación homosexual en Colombia, los discursos de protección y cuidado, es decir, de sentirse invadidos, se activaron únicamente hacia la presencia de un cuerpo trans. Por lo menos en comentarios dirigidos a mí, pues en espacios más privados otros discursos pueden haber aparecido. Lo cual se relaciona con el miedo a la interrupción, que Valeria Flores (2013) reconoce como efecto de complejas 
DOI: http://doi.org/10.46667/renbio.v14i1.458

configuraciones (inter)subjetivas en medio de una profunda economía del afecto y del deseo, que nutre sentimientos como repugnancia, asco, vergüenza y odio. La escuela es un monumento cisgénero, la sola proximidad de sujetos trans a sus ladrillos, especialmente como sujetos de saber, perturba la cisnormatividad. Lo cual se manifestó posteriormente en el inicio de dos procesos institucionales contra mí, que después de mucho apoyo y lucha de colegas y algunos directivos, pudieron resolverse sin alcance mayor.

Ya a nivel de los debates que involucraron argumentos de la biología, la química y el desarrollo de las otras actividades, en los pareceres finales de algunos estudiantes es posible identificar algunos trazos de la violencia objetificadora de la cisnormatividad en los cuales la necesidad de proteger la cisgeneridad se expresa. Quiero ser muy insistente en que esto no debe ser leído como patología o falla moral individual de mis estudiantes, y sí, como expresiones estructurales de violencia, tal vez impulsados y nutridos por las propias características de las actividades de la propuesta didáctica, y todo un histórico educativo. Relaciono algunos a seguir:

Tiffany Abreu tiene el nivel de testosterona menor que el de una mujer normal lo que significa que la que está de verdad en desventaja es Tiffany y aun así eso no ha impedido que Tiffany pueda jugar y ganar partidos de vóleibol, si hablamos por la parte moral Tiffany ha sido juzgada por ser trans y dicen que no puede participar (Fragmento parecer estudiante María)

Pero si se llegara a dar el caso de que llegaran a querer jugar más mujeres trans no se les aceptaría entrar al equipo por que se le perdería el sentido al equipo de voleibol femenino (Fragmento parecer estudiante Carlos)

Ahora por la parte del NO, porque nosotros no sabemos que le pueden hacer sus compañeras o compañeros al momento de jugar, la pueden excluir, rechazar porque simplemente las personas digan que las personas trans tienen una ventaja, o simplemente lo digan porque se sienten superiores a esas personas, en lo cual esto puede herir los sentimientos la persona trans (Fragmento parecer estudiante Martín)

Evidentemente aquí el nivel de violencia es diferente al expresado en las primeras actividades en las cuales se trataba de comentarios entre compañeros de la clase. El parecer final se refería a un trabajo final que sabían tendría autoría declarada y sería leído por el profesor. Estos fragmentos permiten reconocer que cuando abordamos temáticas de identidad de género en la enseñanza de la biología, el proceso de aprendizaje pasará por contradicciones y tensiones entre la violencia objetificadora de la cisnormatividad y posiciones más empáticas y a favor de los derechos humanos. En los fragmentos observamos que en algunos casos se puede apoyar la participación de mujeres trans en equipos femeninos profesionales, utilizando argumentos como los niveles de testosterona, pero aun así diferenciarlas del grupo de "mujeres normales". Asociar que la transfobia existe porque existen personas trans, y no porque existen personas transfóbicas. Proteger a la cisnormatividad de responsabilidades cuando se menciona que mujeres trans no pueden participar de un equipo femenino por la exclusión que pueden sufrir de sus compañeras, como si esa exclusión fuese natural e 
DOI: http://doi.org/10.46667/renbio.v14i1.458

inevitable. $\mathrm{O}$ asumir que solo podría participar una, porque si ese número aumenta se pierde el sentido de lo "femenino".

Más allá de si las y los estudiantes dieron pareceres favorables o no a la participación de Tifanny Abreu en el equipo femenino profesional de voleibol, se observa que la característica de la actividad de colocar personas cisgénero, a pesar de ser una situación ficticia, a decidir sobre lo que puede o no una persona trans, una vez más facilitó la expresión de la defensa a la cisgeneridad y sus trazos de prediscursividad, binariedad y permanencia (VERGUEIRO, 2015) como obligación de los cuerpos, que marca las instituciones deportivas. En ese sentido, se perdió una oportunidad valiosa (por lo menos en la actividad del parecer final) de que las y los estudiantes pudieran reflexionar sobre las propias experiencias de sus cuerpos e identidades, reconociendo las violencias ejercidas por la cisnormatividad también sobre ellos. Siendo este un desafío que se propone desde las transpedagogías, involucrar el propio cuerpo como enunciación de reflexiones éticas para darle fuerza y coherencia a la comprensión de opresiones en la sociedad y las maneras de incidir sobre ellas (LOSADA, 2016).

Estas tensiones y contradicciones fueron reproducidas también por el profesor, familias, las y los estudiantes. No se trata de que unos actuaron positivamente y los otros no. No se trata de un juicio moral o valorativo encasillable en el binario bueno/malo. Tal vez se trata de reconocer que esas contradicciones son propias del proceso de aprendizaje cuando lidiamos con la problematización de estructuras opresivas naturalizadas en las sociedades colonizadas, y que por lo tanto, debemos estar atentas y atentos a estas tensiones para pensar otras propuestas didácticas. Esto también nos indica que estas problematizaciones deben ser permanentes y pensadas a largo plazo. No se resuelven siglos de formación de estructuras opresivas con seis semanas de aplicación de una propuesta didáctica.

Asumir esta discusión históricamente desconsiderada en la biología escolarizada, a pesar de los riesgos de reforzar la violencia objetificadora de la cisnormatividad, abre también oportunidades a la construcción de aprendizajes y reflexiones transgresoras y potentes, especialmente cuando las y los estudiantes pueden dialogar y tener contacto directo con las personas trans como sujetos de saber. A seguir resalto algunas de esas potencias.

\subsection{La influencia y potencia de los saberes trans}

Durante el desarrollo de la propuesta didáctica las y los estudiantes también realizaron reflexiones potentes, especialmente durante las actividades de diálogo directo con especialistas trans sobre la temática, en otras actividades donde sus cuerpos fueron involucrados o en las cuales se realizaron reflexiones críticas sobre estereotipos asociados a las hormonas con la feminidad y masculinidad. En el diario de campo registré algunos recuerdos de una conversación entre estudiantes a partir del análisis del video observado en la actividad 6: 
DOI: http://doi.org/10.46667/renbio.v14i1.458

\begin{abstract}
Durante esta actividad, y corriendo el riesgo de no ser fiel a la conversación, recuerdo que dos estudiantes discutieron los halagos que se hacen a personas cis cuando consumen hormonas con fines deportivos. Fue algo así. -Pablo: "Profe, en ese video dicen que Messi utilizó hormonas para crecer y dicen que está mal, pero de todos modos lo aceptan y a las mujeres trans por qué no al reducir sus hormonas?" -Camilo: "La youtuber lo que critica y dice es que lo aplauden con lo de Messi por ser cis, pero a él le pusieron hormonas para crecer, no para ser mejor" -Pablo: "Pues sí, pero a él lo alaban por como juega, en cambio a Tiffany no" -Camilo: "No, pues la verdad no lo aplaudo por eso, no por las hormonas" Cristian: "Nadie lo criticó porque no afectó la jugabilidad" -Valentina: "Pero si él es más alto puede cabecear más balones ¿no?, entonces sí podría tener ventaja” (Fragmento de registro en diario de campo)
\end{abstract}

Fue el contacto con la mirada de una youtuber trans que permitió que las y los estudiantes retiraran el foco exclusivo sobre el caso de Tiffany haciendo el proceso más relacional, con la problematización del consumo de hormonas en personas cis. Problematizando la idea de naturalidad como marca de la cisgeneridad. Esto fue un paso fundamental para, aunque de manera indirecta, las y los estudiantes reconocieran la cisnormatividad como aquella que coloca como patología cualquier desafío a los trazos de prediscursividad, binariedad y permanencia (VERGUEIRO, 2015), y también como asignadora de privilegios a quien se utilice de estrategias para reforzarlos. Además, la necesidad de traer a la discusión elementos como la nacionalidad y la opresión racista, los cuales se hicieron evidentes al discutir los parámetros que han tomado instancias de poder cuando una situación involucra un deportista sudafricano o uno estadounidense. En estas situaciones, la cisnormatividad se expresa alineada a otras formas de opresión.

Durante la videoconferencia con Ale Mujica, registré en el diario de campo algunas preguntas que mis estudiantes realizaron y que dan cuenta de esa relacionalidad, por ejemplo: “QQué piensan las personas trans de las personas creyentes o muy religiosas?”, “¿En esta sociedad quién mata a las personas trans?", donde respuestas de Ale como: "Simbólicamente, todos los hacemos todos los días", permitió que mis estudiantes y yo generáramos reflexiones sobre nosotros mismos y sobre la cisnormatividad como opresión social.

La actividad 3 también se mostró como una oportunidad para darle vida al cuerpo en el proceso de enseñanza y aprendizaje, problematizando la idea del cuerpo muerto máquina circulante en la biología escolarizada, destacada por Alice Pagan (2018). Trabajar el sistema endocrino no desde los modelos y representaciones fragmentadas de libros didácticos, explorando la posibilidad en siluetas personalizadas de nuestros propios cuerpos. Sobre la actividad de elaboración de las siluetas, registré en el diario:

En $8 B$ fue interesante la actividad de trabajar con las siluetas de sus propios cuerpos. Se mostraron súper interesados en la discusión y en los sistemas endocrino y nervioso. Fue chistoso verles nerviosos, tocándose, haciéndose cosquillas, hombres con hombres, mujeres con mujeres, mientras se dibujaban. Me gustó que sus cuerpos entraron no solo como representaciones a ser observadas en el libro o el tablero, sino también con sus vergüenzas y emociones al dibujarse. Fue interesante, algunos chicos al ver sus siluetas dijeron que tienen cintura de Kim Kardashian y se sorprendieron con las formas de sus cuerpos. Les mencioné que estamos jugando con nuestros cuerpos, de la misma manera que la biología lo hace para generar cuerpos tan diversos que infelizmente la sociedad quiere aprisionar en unos moldes 
DOI: http://doi.org/10.46667/renbio.v14i1.458

específicos y adiestrados para el trabajo, para no manifestar muchas emociones. Les dije que entonces podían jugar con esas representaciones, y algunos de ellos desafiaron lógicas. Por ejemplo, Pedro colocó ovarios en su silueta en lugar de testículos, Ana colocó un ovario y un testículo como sus gónadas porque dijo que había escuchado que es una posibilidad. (Fragmento registro diario de campo)

Involucrar el cuerpo en la enseñanza y del aprendizaje es un elemento fundamental en la propuesta de las transpedagogías, como nuevas poéticas pedagógicas de sanación que "tienen la potencia de habilitar la experiencia de otros cuerpos, otros placeres y otras formas de ver el mundo" (BELLO, 2018, p. 114). O como destaca Lía García (2020), estrategias performáticas, desde el cuerpo y con el cuerpo, para trabajar las situaciones que la propia vida nos va colocando como referentes A los ojos de la cisnormatividad y su trazo de dualidad vigilancia/fragilidad esta actividad puede ser catalogada como un contagio y alimentar el pánico moral de que el profesor quiere que sus estudiantes sean homosexuales o trans (como en medio de un proceso lo mencionó la madre de un estudiante). Sin embargo, Alanis Bello (2018) destaca que en este tipo de actividades:

Este contagio no es el de la homosexualidad ni el del transgenerismo, sino un contagio de la libertad y de la posibilidad de autodeterminación sobre nuestros propios cuerpos, deseos y sexualidades. Esto significa contagiar el purismo de la escuela y sus normas, su obsesión por la mismidad y por la ausencia de conflictos (BELLO, 2018, p. 120)

También en los pareceres finales elaborados por las y los estudiantes sobre el caso de Tiffany, fue posible evidenciar que a pesar de las contradicciones propias de la violencia objetificadora de la cisnormatividad, también hay tensiones generadas por el diálogo con saberes transgresores, por vivencias en la experiencia de enseñanza y aprendizaje que permitieron desafiar los mandatos de la cisnormatividad. Resalto algunos fragmentos:

\begin{abstract}
Aunque biológicamente todos los Seres humanos tanto (Machos y Hembras) poseen en su cuerpo hormonas como la testosterona que pueden ser producidas en testículos, ovarios y glándulas suprarrenales, lo realmente diferente es la variación en la producción que hay de cada una. Lo que verdaderamente potencializa a cada individuo o jugador es su forma en la que se desarrolla y destaca en su entrenamiento [...] Tifanny cuenta con la estética que desea ver la sociedad ("Estereotipos e Imaginarios") que desarrollan para aceptarla en un determinado grupo de la sociedad "Hombre y Mujer" diciéndole como debería ser su forma de actuar, vestir, hablar, pensar, que cosas están bien y que cosas no, etc....., Sin dejar que cada individuo pueda decir lo que quiere para su vida y su futuro [...] Porque lo realmente mal es no dejar expresar, pensar y cuestionar libremente a una persona frente a la perspectiva que quiere ver acerca del mundo y su propio mundo. (Fragmento parecer estudiante Carolina).
\end{abstract}

Tiffany Abreu es una gran representación de que existe una gran diversidad de género, ya que la ciencia compone al cuerpo, y que no existen diferencias sociales ni de habilidades entre personas Cis y Trans....Y como dice un poema: - "Si queremos mudar la política del cuerpo, hay que modificar la ciencia del cuerpo" Pero antes hay que reconocer a la ciencia, pero la duda es ¿Quién hace aquella ciencia? (Fragmento parecer estudiante Tomás) 
DOI: http://doi.org/10.46667/renbio.v14i1.458

Son colocaciones que muestran que es posible abrir brechas, tensionar pensamientos y estereotipos solidificados y hasta fortalecidos en imaginarios de legitimidad científica. Que lo propio de la biología es la diversidad de posibilidades, y no una indisolubilidad con la cisnormatividad. En las colocaciones del estudiante Tomás se hace una mención explícita a la ciencia y su papel en las relaciones de poder que favorecen la cisnormatividad "¿Quién hace aquella ciencia?" lo cual fue una influencia directa del diálogo con Ale Mujica, quien trajo esta discusión y a quien el estudiante mencionó en su parecer. De otra parte, las colocaciones de la estudiante Carolina reflejan un acercamiento a la comprensión (de manera implícita) de la cisnormatividad como norma de la sociedad que impone "estereotipos e imaginarios" de masculinidad y feminidad, reconociendo que el problema no es la diversidad, y sí, la normativa social que no permite "expresar, pensar y cuestionar libremente a una persona frente a la perspectiva que quiere ver acerca del mundo y su propio mundo". Estos procesos pedagógicos y didácticos podrían ser de larga duración y extenderse a la enseñanza de otros contenidos, pues quizás la potencia de estos abordajes no está en las respuestas acabadas, y sí, en la posibilidad de vivir estas contradicciones y tensionamientos permanentemente.

\section{Consideraciones finales}

Con este trabajo se evidenció que las clases de biología pueden ser territorios donde la violencia objetificadora de la cisnormatividad puede expresarse y reforzarse, inclusive, en el desarrollo de propuestas didácticas que pueden considerase progresistas o transgresoras desde los referentes teóricos actualmente discutidos en el campo. Estas violencias se reproducen en medio de tensiones con brechas, fugas y desestabilizaciones permitidas por los diálogos de los sujetos que participan del proceso de enseñanza y aprendizaje con los saberes, luchas y demandas de las personas trans como sujetos de saber. El trabajo permite reflexionar sobre la necesidad de que la problematización de la cisnormatividad ocupe un lugar más protagónico y explícito en las incursiones de género y sexualidad en la biología escolarizada. Considerando además que a nivel educativo y de investigación, aún la enseñanza de la biología es un espacio dominado en su gran mayoría por personas cisgénero.

Es importante que los resultados aquí presentados nos estimulen a pensar otros caminos, sin desvincularnos totalmente de un sentir comprensivo de la realidad de profesoras y profesores de biología en el escenario actual de avance neoliberal y fascista, donde muchos impases económicos, políticos e institucionales surgen con estos abordajes. Este trabajo no tiene como propósito colocar una obligación moral más a las y los profesores que ya tenemos que trabajar en contextos muchas veces adversos y precarios. El objetivo fue señalar una tensión, contradicción propia del proceso de enseñanza y aprendizaje, que hasta ahora no había sido referenciada, debido a la ausencia de trabajos sistematizando desde la realidad de la escuela, los impactos de propuestas didácticas comprendidas como progresistas dentro del campo. Tal vez considerar esta tensión pueda permitirnos pensar otros caminos que no refuercen la violencia objetificadora de la cisnormatividad. 
DOI: http://doi.org/10.46667/renbio.v14i1.458

Las personas trans y cis somos más que hormonas, genética y sistema endocrino. Más allá de pensar cómo incluimos cuerpos trans en la enseñanza de la biología (y la química), parece más relevante pensar las contribuciones de las transpedagogías (LOSADA, 2016; BELLO, 2018; PAGAN, 2018) y las epistemologías, saberes trans para pensar todo el proceso pedagógico y didáctico, así como nuestras sensaciones sobre la naturaleza, sobre la sociedad y sobre nosotros mismos.

\section{Agradecimientos}

Algunas reflexiones y conceptualizaciones aquí presentadas fueron producto del diálogo cariñoso y crítico con el intelectual, activista trans y Magister en Estudios de la Cultura, Camilo Losada, al socializarle informalmente la experiencia. Infinitas Gracias a él y al Dre. Ale Mujica por las oportunidades de aprendizaje y ricas reflexiones. Agradecimientos a CAPES por el auxilio financiero para el desarrrollo de la investigación.

\section{Referencias}

BELLO, Alanis. Hacia una trans-pedagogía: reflexiones educativas para incomodar, sanar y construir comunidad. Debate feminista, v. 55, p. 104-128, 2018.

FARIAS, Yaci. "Uma canção pra você" a música em uma proposta de sequência didática voltada para um ensino de biologia não sexista. Revista Ensino de biologia, v. 13, n. 2, p. 268-288, 2020.

FLORES, Valeria. Interrupciones ensayos de poética activista escritura, política, educación. Neuquen: La Mondonga Dark, 2013.

GARCIA, Ketlyn; LOPES, César; LOGUERCIO, Rochele. Trans-orgânica uma proposta de ensino de química orgânica utilizando as temáticas de gênero e sexualidade. In: CONGRESO

INTERNACIONAL SOBRE INVESTIGACIÓN EN DIDÁCTICA DE LAS CIENCIAS, 10, 2017

Memorias... Sevilla, España, 2017.

GARCÍA, Lía. El deseo, el afecto y la seducción como categorías pedagógicas. Entrevista realizada por Diego Falconi. Revista Interdisciplinaria de Estudios de Género de El Colegio de México, v. 6, e549, 2020.

GRAHAM, Andrew. Como escrever e usar estudos de caso para ensino e aprendizagem no setor público. Brasilia, DF: ENAP, 2010.

LOSADA, Camilo. Pedagogías decoloniales y cocuidado: un aporte en la reconstrucción y restitución de la memoria colectiva de hombres transgénero de la organización social hombres en desorden. Trabajo de conclusión de curso (Licenciatura en Educación Comunitaria) - Universidad Pedagógica Nacional. 2016.

MARIN, Yonier; NUNES, Pamela; CASSIANI, Suzani. A Branquitude e a Cisgeneridade problematizadas na formação de professoras(es) de Ciências e Biologia: uma proposta decolonial no estágio supervisionado. Ensino, Saúde e Ambiente - Número Especial Educação Ambiental: Insurgências, Re-Existências e Esperanças, p. 225-238, 2020.

MARIN, Yonier; OLIVEIRA, Maira. Problematizando as relações entre química-biologia e questões de gênero: possibilidades e desafios na educação de jovens e adultos. Revista Debates em Ensino de Química, v. 5, n. 2, p. 19-38, 2019. 
DOI: http://doi.org/10.46667/renbio.v14i1.458

MARIN, Yonier. Problematizando el discurso biológico sobre el cuerpo y género, y su influencia en las prácticas de enseñanza de la biología. Revista Estudos Feministas, v. 27, n. 3, p. 1-10, 2019. https://doi.org/10.1590/1806-9584-2019V27N356283

MENDEZ, Alexis. La perspectiva de género en la enseñanza del sistema endocrino: una experiencia didáctica en contexto de educación remota. Revista de Educación en Biología, n.esp., p.12, 2020.

NASCIMENTO, Caroline. Educação das relações étnico-raciais: branquitude e educação das ciências. Tese (Doutorado em Educação Cientifica e Tecnológica) - Universidade Federal de Santa Catarina, 2020.

PAGAN, Alice. Entre o bélico e o diplomático: transicionar a ciência como possibilidade de humanizar a educação ambiental. Revista Sergipana de Educação ambiental, v. 7, p. 1-19, 2020.

PAGAN, Alice. O ser humano do ensino de biologia: uma abordagem fundamentada no autoconhecimento. Revista entreideias, v. 7, n. esp., p. 73-86, 2018.

PONTES, Ana; NETO, José. A fada madrinha da passabilidade: hormônios e o ensino de química. In: OLIVEIRA, Roberto; QUEIROZ, Gloria. (Org.) Conteúdos cordiais: química humanizada para uma escola sem mordaça. São Paulo: Editorial da Física, 2017.

RANNIERY, Thiago. Gênero não tem cabimento, nem nunca terá: ensino de biologia e a relação natureza e cultura. Revista educação e cultura contemporânea, v. 18, n. 52, p. 485-516, 2021.

TAVARES, Bruno. Cisheteronormatividade no livro didático de biologia: análise à luz da pedagogia queer. In: Guimaraes, R. S.; VERGUEIRO, V.; MARCOS, M.A.; FORTUNATO, I. (Org.). Gênero e cultura: perspectivas formativas; vol. 2. São Paulo: Edições Hipótese, 2018.

SANTOS, Sandro; QUEIROZ, Elenita. Ensino de biologia e transsexualidade. Ensino em Revista, v. 26, n. 1, p. 147-172, 2019.

SANTOS, Sandro; MARTINS, Matheus. Entre encontros e ensino de biologia e gêneros e sexualidades: sopros e insurgências de uma biologia menor. REnBio, v. 13, n. 1, p. 141-152, 2020.

SANTOS, Silvio. O método da autoetnografia na pesquisa sociológica: atores, perspectivas e desafios. Plural, v. 24, n. 1, p. 214-241, 2017.

VERGUEIRO, Viviane. Por inflexões decoloniais de corpos e identidades de gênero inconformes: uma análise autoetnográfica da cisgeneridade como normatividade. Dissertação (Mestrado em Cultura e Sociedade) - Universidade Federal da Bahia, 2015.

WAYAR, Marlene. La visibilidad de lo invisible. In: BERKINS, Lohana. Cumbia, copeteo y lágrimas: informe nacional sobre la situación de las travestis, transexuales y transgéneros.. Buenos Aires: A.L.I.T.T. Asociación de lucha por la identidad travesti-transexual. 2007.

YORK, Sara; OLIVEIRA, Megg; BENEVIDES, Bruna. Manifestações textuais (insubmissas) travesti. Estudos feministas, v. 28, n. 3, e75614, 2020.

Recebido em fevereiro de 2021. Aprovado em abril de 2021.

Revisão gramatical realizada por: Dre. Ale Mujica Rodriguez

E-mail: mujica.rodriguez86@gmail.com 